\title{
Transitory selection markers facilitate DNA mutagenesis with recombineering
}

\author{
J rGang Cheng, Steven M. Hollenback, Yaohong Wu, Vladimir Ghukasyan, Suzanne Lyman \\ UNC Neuroscience Center, UNC-Chapel Hill, Chapel Hill, NC, USA.
}

Correspondence: J rGang Cheng. Address: UNC Neuroscience Center, NSRB\#7119A, 115 Mason Farm Road, UNC-Chapel Hill, Chapel Hill, NC 27599-7250, USA. Email: jgc5123@gmail.com

Received: October 28, 2015

Accepted: December 6, 2015 Online Published: December 16, 2015

DOI : $10.5430 /$ jbei.v2n2p23

URL: http://dx.doi.org/10.5430/jbei.v2n2p23

\begin{abstract}
Site-directed mutagenesis with DNA oligos is a powerful tool to generate subtle alterations in DNA. Taking advantage of strand replacement during lambda-red recombineering, mutations on DNA (donor) can be introduced into another DNA construct (acceptor) via targeting. We describe a novel approach that takes advantage of the specificity of homologous recombination and the convenience of positive selection to insert mutations into existing DNA constructs. With simple procedures, we generated a "transitory" selection marker that is used to carry modifications into acceptor DNA and then removed by enzyme manipulation, leaving only the desired mutation without any scar. Overall, this method of targeting mutagenesis via recombineering provides a precision manipulation of DNA and avoids the extensive PCR reaction. Not only can this method be used to make point mutations, truncations or Epitope-tags, but it can also be used to import existing mutations, generate in-frame fusion, or repair missing components.
\end{abstract}

\section{Key words}

Recombineering, $\lambda$-Red, Site-directed mutagenesis, In-frame fusion

\section{I ntroduction}

Since its invention in the 1970s, site-directed mutagenesis, which provides a practical way to modify existing DNA molecules, has had a profound impact in biomedical research ${ }^{[1]}$. Many improvements and modifications have been developed to simplify the original approaches and reduce the background, especially with the advance of high fidelity PCR (HF-PCR) and long-extend PCR. Furthermore, utilizing DNA synthesis, mutations can be introduced to DNA by combining several complementary and overlapping long oligos. With the exception of natural occurrence mutations, in most cases the DNA oligo is a crucial component of site-directed mutagenesis. Desired mutations can be incorporated during DNA oligo synthesis, which may be used as a primer for DNA replication or PCR amplification to generate DNA fragments with mutations.

Whether it is a mutation formed by oligo or the existing DNA mutation, the mutated DNA fragments need to be incorporated into a vector for replication. While mutated primers can be incorporated into a plasmid by DNA replication, PCR-generated mutations of DNA fragments may be ligated to a vector. The challenge lies in how to distinguish the mutated DNA from parental plasmids in a manner that can be easily identified. Traditionally, parental plasmids can either grown in a bacteria strain that inserts UTP to replace dTTP, or alternatively, the DNA is methylated ${ }^{[2,3]}$. After the plasmid 
is primed or PCR amplified with a mutated oligo, the resulting hybrid plasmids with UTP or methylated DNA can be respectively degraded by DNA repair or by using a methylation-specific restriction endonuclease (RE) such as DpnI ${ }^{[4]}$. Alternatively, to reduce the background and undesired alterations of the plasmid, the modified DNA fragments can be subcloned and processed independently before being ligated back into the final vector. In this event, one or two RE sites, preferably unique, are needed to shuttle DNA between vectors. Finally, DNA sequencing is used to confirm the generation of the desired mutation.

With the adaptation of homologous recombination (HR) in DNA manipulation (recombineering), the procedures of site-directed mutagenesis can be modified and used to generate mutations in large DNA constructs such as Bacterial Artificial Chromosome (BAC) ${ }^{[5-8]}$ or high copy plasmids ${ }^{[9]}$. The widely used Lambda red operon provides three inducible enzyme components to facilitate HR during DNA replication ${ }^{[10]}$. With as few as 30 50 nucleotides of each homologous arm, DNA can be altered through recombination to bring insertion mutations into the DNA of plasmids or bacterial chromosomes. With positive selection markers included in the targeting cassette, targeted DNA can be sorted out with antibiotic selection to reduce the effects of PCR or DNA sequencing screening. However, to generate a mutation without the addition of an extra-DNA sequence (scar-free), special tools such as counter-selection or extensive screening with PCR reactions are needed ${ }^{[11]}$. Still, counter-selection, although convenient, has limitations. First, special care is required to maintain its potency. Secondly, specific conditions such as the bacterial strain (rpsl, galk) or specific substrate (SacB-sucrose, GalK-glucose \& TetR-fusaric acid) need to be matched ${ }^{[12,13]}$. Finally, an additional targeting step is required to remove the selection marker. Furthermore, it is usually not suitable to utilize counter-selection with smaller plasmids with medium to high copy numbers.

With the increased size and complexity of modern day vectors, traditional site-directed mutagenesis approaches are insufficient to handle such tasks. Novel approaches such as Gibson assembly require multiple rounds of PCR and proof of DNA sequencing without error ${ }^{[14]}$. To modify medium size plasmids between small plasmids (which are PCR manageable) and large and low copy number plasmids (which rely on counter-selection and multiple targeting), it is therefore preferable to employ recombineering with positive selection only. We acquired the desired mutations in DNA between homologous arms (for insertion) as well as part of homologous arms (for deletion and point mutation) and then introduced them into targeted DNA via strand replacement during DNA replication ${ }^{[15]}$. Combining this character with the transitory selection marker (tSM), the positive SM used in the targeting cassette can be removed seamlessly without any scar through enzyme manipulation. Except for the desired mutation, the resulting plasmids are identical to those of the parental plasmids. Such targeting mutagenesis by recombineering (recombimutagenesis) has been adapted to bring in point mutations, peptide tags, as well as in-frame fusion between proteins. Our approach simplifies the effort of performing site-directed mutagenesis, reduces the implementation of long PCR reactions, decreases the screening effort, and provides an additional tool for DNA/protein engineering.

\section{Methods}

\subsection{Material}

Recombineering plasmid pKD46 is from Dr. B. Wanner at Purdue though CGSC (E. coli Genetic Stock Center) in Yale. Recombinogenic bacteria strains and em7Neo are from Dr. N. Copeland of NCI at Frederick. GFP-Cre and GFP-CreERT2 and Myr-tagRFP-T plasmids are from Dr. A. McMahon of Harvard. All restriction endonuclease and most enzymes are from NEB (New England Biolabs).

\subsection{Recombineering}

Details of the protocol using DY380 ${ }^{[16]}$ and pKD46 ${ }^{[17]}$ can be found in the cited references. In short, to make a plasmid targetable, an acceptor plasmid was sent into a recombinogenic bacteria strain such as DY380 under the selection of a 
plasmid's own antibiotic resistance and tetracycline $(12.5 \mu \mathrm{g} / \mathrm{ml})$. Alternatively, pKD46, which contains an Arabinoseinducible Lambda red operon, was sent into the host of the acceptor plasmid. Electroporation (Eppendorf, Electroporator $1660 \mathrm{~V}$ ) was used in all the transformations after the bacteria cells were washed with ice-cold water. In order to circumvent the antibiotic resistant conflict of $\mathrm{pKD} 46$, which is AmpR and shared with many popular plasmids, we swapped the AmpR coding region of pKD46 with other selections such as Kan, CM, Zeo or Hygro via targeting. Both DY380 and pKD46containing bacteria are temperature-sensitive, and cultured at $32^{\circ} \mathrm{C}$.

Recombinogenic bacteria was treated with a temperature shift to $42^{\circ} \mathrm{C}$ for $15 \mathrm{~min}$ (DY380) or inoculated with $0.1 \%$ Arabinose for 1 to 3 hours (pKD46) to induce Lambda red activities. Then treated cells were made electro-competent, mixed with a prepared targeting cassette, and targeting occurred after electroporation in a $1 \mathrm{~mm}$ cuvette. Bacteria was flushed out with $0.3 \mathrm{ml} \mathrm{SOC}$ and incubated at $32^{\circ} \mathrm{C}$ for an hour. $1 / 10$ of the SOC culture was plated on an agar-plate with selections against both acceptor plasmid and targeting cassettes. In order to remove Lambda red activities, targeted plasmids can be retransformed to new cells or pKD46 can be cured while growing bacteria at $37^{\circ} \mathrm{C}$ to $42^{\circ} \mathrm{C} \mathrm{O} / \mathrm{N}$.

\subsection{Generating mutated DNA and targeting cassettes}

Primers used in each experiments are listed in Table 1, which usually containing desired mutations, a unique RE site and a SM-specific sequence that can be used to generate targeting cassettes by HF-PCR with SM as a template. Alternatively, DNA containing existing mutations, or de novo mutations generated by OLPCR can be cloned into TA vectors for sequence confirmation of the desired mutation. A selection marker flanked by a unique RE site was then added via ligation or targeting to make the mutated DNA as a targeting cassette.

To make GSK3 $\beta$ targeting cassettes with a point mutation, primer L to pair with pM-Gly or pM-Ala and primer R to pair with pO-Gly or pO-Ala were used to amplify the products L/M and O/R with HF-PCR with Phusion polymerase (NEB M0530). There is a 12 bp overlap between these two PCR products. After purification of each product with agarose gel, the same ratio of PCR products was added as a template and an overlapping PCR was performed in which primer $\mathrm{L}$ and $\mathrm{R}$ were added after 5 cycle of self-extension at $37^{\circ} \mathrm{C}$ before resuming the standard HF-PCR reaction. The PCR product with the correct size was cloned into a TA vector (pGEMTEasy, Promega) and DNA sequencing confirmed the correct mutation. TA cloned Plasmid-pL/R was digested with BspE1 and ligated to BspE1 flanking RPSL-Neo, which was generated by using primers BspE1RV-RLNeoF and BspE1RV-RLNeoR with RPSL-Neo as template. Either NotI fragment or HF-PCR product with primers L/R was used to generate a targeting cassette. To reduce false positives, DpnI was added to remove plasmid templates, and DraI, BspHI, ScaI or XmnI was used to degrade vector backbones.

Similar procedures were used to generate the targeting cassettes for the Ampk $\gamma$ point mutation (CGA $\rightarrow$ CAA) with primers $\mathrm{pL} / \mathrm{pM}$ and $\mathrm{pO} / \mathrm{pR}$. A G $>$ A mutation resided within 21 base pairs of the overlapping parts. After TA cloning of PCR products from the OLPCR reaction, DNA sequences were used to confirm the desired mutation. The correct plasmid was digested with StuI and ligated to AscI flanked Neo, which was digested with AscI and filled in with Klenow reaction. Either a NotI fragment or HF-PCR product with primers L/R were used to generate targeting cassettes.

To generate targeting cassettes for 4 mutations of mFMR1, fragment of em7Zeo (from pCMV-Zeo, Invitrogen) was used as template and PCR with each pair of long primers LA/RA that provide homologous arms, mutation (R138Q/ G481S/S499D/R533H), unique site (NheI/ BtmI) and identical SM specific primer (Em7F and ZeoCR).

Self-splicing peptide F2a contains 24 amino acids and 72 bps. HFPCR with primers containing half of the F2a sequence, FseI and either em7 forward or Neo reverse sequence (F2a-FseI-em7F and F2a-FseI-NeoR) were used to generate F2aFse-em7-Neo cassettes. Then F2a-Fse-em7-Neo served as template to generate targeting cassettes by adding homologous arms against GFP and Cre with LAGFPC-F2aF and RACreN-F2aR. 
Table 1. Primers sequences

\begin{tabular}{|c|c|}
\hline GSK3 $3-p L$ & 5' TGGTAGGTGCATTTGTTCAGCTC 3' \\
\hline GSK3 $\beta-p M-G l y$ & 5' gtcgccCACCAGGTTAAGG TAGACCTCATCTTTC 3' \\
\hline GSK3 $\beta$-pM-Ala & 5' gtcggcCACCAGGTTAAGGTAGACCTCATCTTTC 3' \\
\hline GSK3ß-pO-Gly & 5' CTTAACCTGGTGGGCGACTATGTTC 3' \\
\hline GSK3ß-pO-Ala & 5' CTTAACCTGGTGGCCGACTATGTTC 3' \\
\hline GSK3 $\beta-p R$ & 5' TTCTGGAAACTAGAAGGGGTTCG 3' \\
\hline BspE1RVRLNeoF & 5' ATATCCGGATATCGGCCTGGTGATGATGGCGGGATCG 3' \\
\hline BspE1RVRLNeoR & 5' ATATCCGGATATCAGAAGAACTCGTCAAGAAGGCG 3' \\
\hline Ampk $\gamma-p L$ & 5' ACTGCGAGGGGATGGTACTTTG 3' \\
\hline Ampk $\gamma-p M$ & 5' GCGGTGCCGCTÍTCACACCGTTGTAGACGAGGGCGTAGAAG 3' \\
\hline Ampk $\gamma-\mathrm{pO}$ & 5' ACGGTGTGCAㅁGCGGCACCGCTCTGGGATTCGGAGAAGCAAC 3' \\
\hline Ampk $\gamma-p R$ & 5' TTTGATGGCATCGTAGAGGGAC 3' \\
\hline em7F & 5' TGTTGACAATTAATCATCGGCA 3 \\
\hline ZeoCR & 5' TCAGTCCTGCTCCTCGG 3' \\
\hline mFMR1R138QLA & 5' TCAAGCTGGAGGTGCCAGAAGATTTAcagCAAATGTGTGctagc-em7F 3' \\
\hline mFMR1R138QRA & 5' GCCTTTTTAAAATCCTTATGTGCTGATTCTTTGGctagc-ZeoCR 3' \\
\hline mFMR1G418SLA & 5' CGAGGTAGTAGACCTTACAGAAATAGGGGGctagc-em7F 3' \\
\hline mFMR1G418SRA & 5' AATTAGTTCCTGAAGTATATCCAGGACCGCGTCTagaGTGctagc-ZeoCR 3' \\
\hline mFMR1S499DLA & 5' TACTTCAGGAACTAATTCTGAAGCATCAAATGctagc-Em7F 3' \\
\hline mFMR1S499DRA & 5' CACTGAGTTCGTCTCTGTGGTCAGATTCTGTTTCgtcAGctagc-ZeoCR 3' \\
\hline mFMR1R533HLA & 5' GGGAGAGCTTCCTGCGCAGAGGAGACGGACGGctagc-em7F 3' \\
\hline mFMR1R533HRA & 5' ССТСТTCСТCСТTGTCСТCTTCСТCСТCСТCCgtgCCGctagc-ZeoCR 3' \\
\hline F2a-FseI-em7F & $\begin{array}{l}\text { 5' GCACCGGTGAAACAGACTTTGAATTTTGACCTTCTCAAGTTGGCCG } \\
\text { GCCGACCTGCAGCCTGTTGA 3' }\end{array}$ \\
\hline F2a-FseI-NeoR & 5' GGGCCCTGGGTTGGACTCCACGTCTCCGGCCGGCCTCAGAAGAACT CGTCAAGAAG 3' \\
\hline LAGFPC-F2aF & $\begin{array}{l}\text { 5' GTACAGCTCTCGACGGAGAAAGCTCAGGCTCTGGCTCAGAGTCTGA } \\
\text { CTCCGCACCGGTGAAACAGACTTTGAA 3' }\end{array}$ \\
\hline RACreN-F2aR & $\begin{array}{l}\text { 5' TCGACCGGTAATGCAGGCAAATTTTGGTGTACGGTCAGTAAATTGG } \\
\text { CCATGGGCCCTGGGTTGGACTC 3, }\end{array}$ \\
\hline Rb-bGHpAF & 5' CCCCTGCTGTCCATTCCTTATTC 3’' \\
\hline SV40ProR & 5' GGTTGCTGACTAATTGAGATGCG 3' \\
\hline
\end{tabular}

\subsection{Removal of selection markers}

After targeting and the desired mutation were introduced into the acceptor plasmids, selection markers were removed with enzyme sites that flank the SM. The extra nucleotides, which were added to generate a unique site, were then removed to restore CDS. To polish 3' overhangs, standard Klenow or T4 polymerase reaction was used with the supplement of $1 \mu 1$ of $10 \mathrm{mM}$ dNTPs either by adding $1 \mu \mathrm{l}$ Klenow into the RE mixture and incubating at RT for 30 min or through the use of a Quick Blunting Kit (NEB E1201) ${ }^{[18]}$. To blunt 5 overhangs, 2.5 20 units of Mung Bean Nuclease (NEB M0250) were used and followed by phenol / chloroform extraction and EtOH precipitation to remove enzymes. PCR was then performed on the ligation product and confirmed the change of restriction sites. Blunted DNA was then ligated and transformed into NEB 10-beta cells (NEB C3019H). Individual colonies were checked through evaluation of the loss of antibiotic resistance and RE digestion of the PCR products around targeted site. 


\section{5 $2 \mathrm{~A}$ peptide assay}

Expression constructs (pCAG-Cre-IRES-eGFP, pCAG-eGFP-Cre and pCAG-eGFP-F2a-Cre) by themselves or together with pCAG-STOP-myr-tagRFP-T were transfected into NIH3T3 cells that were cultured in DMEM containing $10 \%$ calf serum. The day prior to the transfection, cells were seeded in 24 well plates so that they were $80 \%$ confluent at the time of transfection. $3 \mu \mathrm{l}$ of Lipofectin (LF2000, Invitrogen) was mixed with $47 \mu \mathrm{l}$ OptiMEM and incubated at room temperature for 5 minutes. $800 \mathrm{ng}$ DNA was mixed in a total volume of $50 \mu \mathrm{l}$ OptiMEM. The LF2000 solution was mixed with the DNA and allowed to incubate at room temperature for 20 minutes. The media was removed from the well and $0.5 \mathrm{ml}$ of fresh media was added. The $100 \mu 1$ of DNA complexes was added to the cells and allowed to incubate for 4-6 hours before changing the media.

For co-transfection experiments, all volumes were doubled and $800 \mathrm{ng}$ of each plasmid was used. After 24 hours, fluorescent cells were easily visible. The cells were lifted from the wells by trypsinization, and washed once to remove the trypsin. A portion of the cells was reseeded on circular poly-D-lysine-coated coverslips and the remaining cells were subjected to be lysed for protein blot. After 24 hours, the coverslips were rinsed once with PBS, fixed in 4\% paraformaldehyde in PBS for 15 minutes, rinsed again and mounted in Vectashield (with DAPI, Vector Lab) on glass slides. Imaging was performed on the Olympus FV1000 confocal microscope.

A

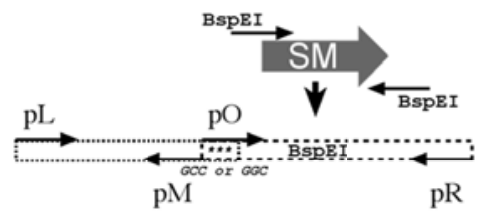

C

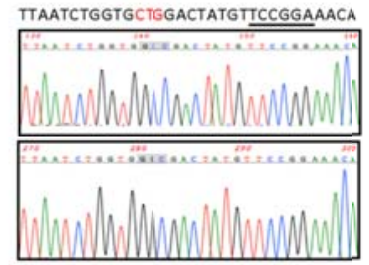

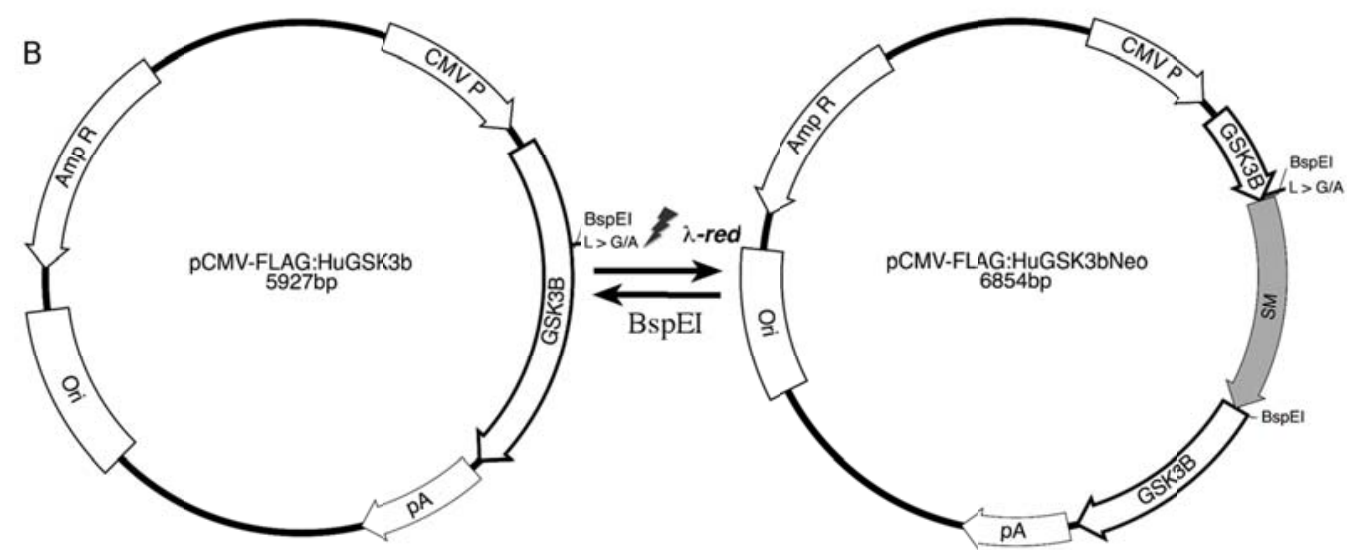

Figure 1. Converting point mutation with targeting. A) Assembly of targeting cassette: Overlapping PCR (OL-PCR) was used to generate mutations with indication of primers' location ( $\mathrm{pL}, \mathrm{pM}, \mathrm{pO}$ and $\mathrm{pR}$ ). PCR generated SM (RPSL-Neo) with primers that contains BspEI sites, was digested with BspEI and inserted into same enzyme site within OL-PCR DNA after TA cloning. B) Map of plasmids: Acceptor plasmid (pCMV-FLAG: HuGSK3b) with marked mutation (L $>$ G/A [Leu $>$ Gly or Ala]) and BspEI sites were altered after targeting (electroporation [lightning] and $\lambda$-red induction) to become targeted plasmid (pCMV-FLAG:HuGSK3bNeo), and reversed to acceptor plasmid but mutations after BspEI digestion. C) Alignment: Nucleotides around targeted site with original sequence and mutation. DNA sequence tracks show the alteration of nucleotides from CTG to become GGC or GCC. BspEI site is underlined. 


\section{Results}

\subsection{Targeting imports mutation}

To establish that targeting can insert both selection markers and desired mutations in a site-specific manner (especially mutation with a few nucleotides within a homologous arm), we made two different point mutations in the same location within the hGSK3 $\beta$ expression vector (pCMV-FLAG-hGSK3 $\beta$, see Figure 1A). GSK3 $\beta$ is an essential signaling component for several signaling pathways. Based on the approaches of pharmacological genetics, it is possible to introduce a mutation in GSK3 $\beta$ that confers selectivity to the modified PPI analogs that do not inhibit WT GSK3 $\beta^{[19]}$. This is accomplished by changing the gatekeeper amino-acid leucine 131 in the ATP binding pocket of the human GSK3 $\beta$ protein to either glycine or alanine.

Overlapping PCR was performed with hGSK3 $\beta$ plasmid as template to make L (CTG) to G (GGC) or A (GCC) mutations ${ }^{[20]}$. After performing TA cloning of the PCR product, mutations were confirmed by DNA sequencing. To generate the targeting cassette, a unique BspEI site $11 \mathrm{bp}$ downstream of the mutation site within the mutated GSK3 $\beta$ PCR products was used to ligate with a BspEI flanked selection fragment (RPSL-Neo) (see Figure 1B). After recombineering, the desired mutation in targeted plasmids were confirmed by DNA sequencing (4 of 4). BspEI was then used to remove the SM, followed by self-ligation and transformation, and the open reading frame of GSK3 $\beta$ was restored (see Figure 1C).

A

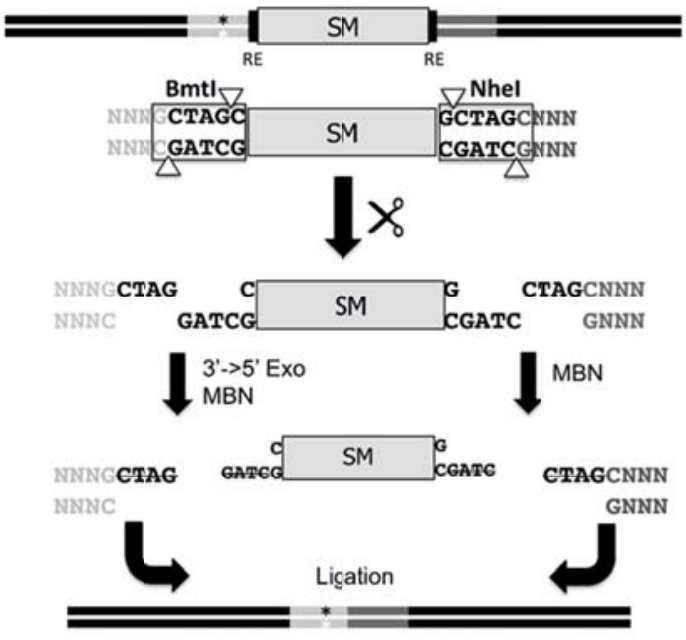

B

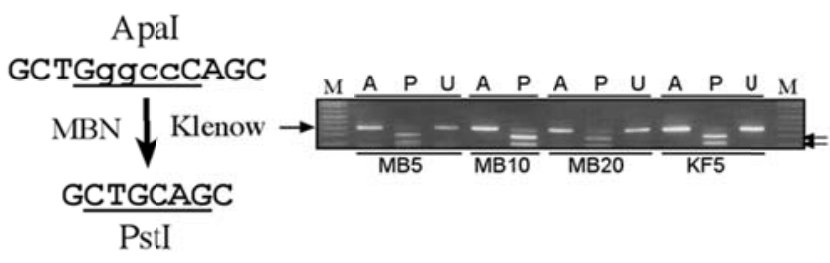

HindIII

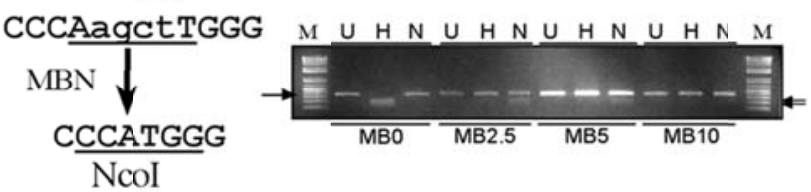

Figure 2. Making transitory selection marker. A) Zero sum design: Diagram shows the principle of making a SM, not only removable but also scarless, by flanked it with a pair unique enzyme sites that can only be used to remove SM but as a non-cutter of an acceptor plasmid. After digested with such enzymes to remove SM, it might either generate a 5' overhangs (NheI) or 3' overhangs (BtmI). Those overhangs can then be flushed with Mung Bean Nuclease (MBN, 5' and 3' overhang) or 3' $>$ 5' exonuclease such as Klenow Fragment (3' overhang) to restore original sequence. B) Proof of concept: Using suitable DNA sequence with enzyme titration, we tested both 3' overhang flushing (pCZV-bGHpA, GCTGggce*CAGC-; ApaI GGGCC*C > PstI CTGCAG) and 5' overhang flushing (pCMV-DsRed, -CCCA*agctTGGC-; HindIII A*AGCTT > NcoI CCATGG). Digested PCR products indicate the site change, residues full-length PCR products, indicated a non-ideal flush (Left arrows, full length; Right arrows, digested). Plasmid was digested with original enzyme (A:ApaI or H:HindIII) and blunted with MBN (or Klenow), and ligated. Using primers pairs flanking enzyme site Rb-bGHpAF and SV40ProR for pCMV-DsRed; pUCOri and bGHpAR for pCZV-bGHpA Resulting PCR products were digested with both old (A:ApaI or H:HindIII) and new (P:PstI or N:NcoI) enzymes. U (uncut) > A or P (416 bp > 204/212 bp): U > H or N (411 bp > 134 / 277 bp). 


\subsection{Transitory selection marker}

It is a rare occurrence that a unique site is near a desired mutation. To circumvent such limitations, a removable and "scarless" transitory selection marker (tSM) in desired location is necessary and clearly advantageous. In conjunction with blunting reactions, certain RE sites, which could yield sticky ends after digestion, can be added and then removed completely together with flanked SM (see Figure 2A). In principle, a tetra-nucleotide palindrome can add 1 bp to become a penta-nucleotide, 2 bp to become a hexa-nucleotide, or 4 bp to become an 8-bp enzyme cutter. A complementary pair of nucleotides can be modified to become 6 cutters with 4 nucleotide extensions. In practice, a penta-nucleotide is hardly unique, and is also rarely to be used. Thus, only 6 cutters to 8 cutters are considered.

Suitable RE sites derived from non-cutters can be added to PCR primers, formed by ligation or by targeting. Flanked by a pair of such "unique" RE sites, SM can be easily removed with enzyme(s) that recognize those sites. Afterwards, the leftover nucleotides from enzyme digestion can be removed by a blunting enzyme. The 3' $>5$ ' exonuclease activity of polymerases, such as DNA polymerase I (Klenow fragment) or T4 DNA polymerase, can be used effectively to flush 3' overhangs ${ }^{[18]}$. Only mung bean nuclease $(\mathrm{MBN})$ can be used to remove a single strand DNA with either 3' or $5^{\text {' }}$ overhangs ${ }^{[21]}$.

A
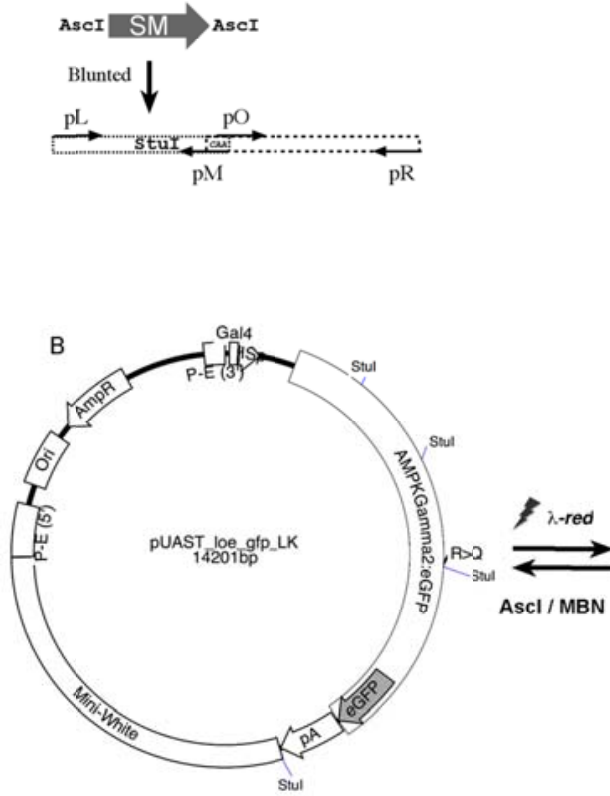

c
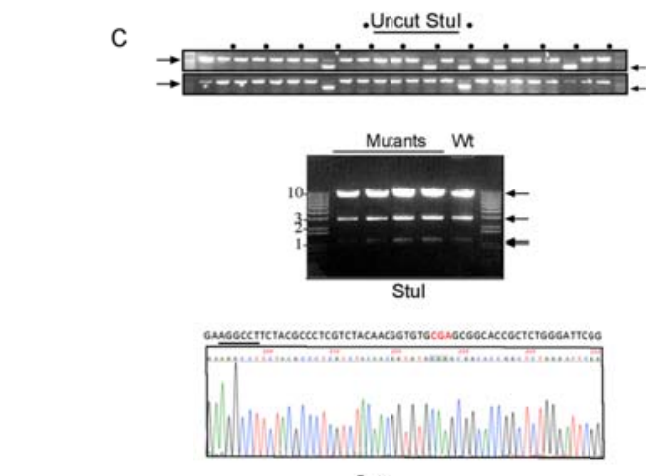

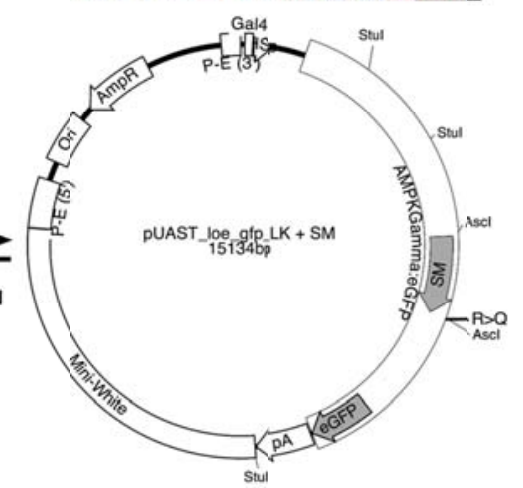

Figure 3. Forming unique site to facilitate tSM insertion and targeting. A) Assembly of targeting cassette: OL-PCR was used to generate point mutation (CAA) with indication of primers' location, AscI flanked SM (Neo) was excised from another plasmid and inserted into StuI with blunted end ligation. B) Map of plasmids: Acceptor plasmid (pUAST_loe_gfp _LK) with indication of mutation $(\mathrm{R}>\mathrm{Q})$ and multiple StuI sites. After targeting, SM and mutation were inserted resulting in the loss of one of the StuI sites and generating two AscI sites that flank SM (pUAST_loe_gfp_LK + SM). After AscI digestion and MBN treatment, StuI restored and reversed to original plasmids with mutations. C) Screening and alignment: A similar approach with MBN flushing as in Figure 2B was used to address the efficacy of MBN treatment (Data not shown), as restoring StuI can be easily assayed after PCR out the flanking region of the mutation with primer L /R. Six of 24 individual colonies were able to be digested with StuI, indicating an ideal flushed ends (Left arrows, full length; Right arrows, digested). All the mutants shared similar digestion patterns as the original one (WT [9.3, 2.8, 1, 1.1.0 kb]). The DNA sequencing track shows the alteration of nucleotides and the restoration of the StuI site (underline). 
The drawback of MBN is that it is difficult to inactivate and less effective in removing of $\mathrm{G}$ or $\mathrm{C}$ overhangs. It also has the tendency to remove extra-nucleotides of T if "breathing" of the DNA ends takes place ${ }^{[2]}$. In addition, AT rich areas within DNA can be digested by MBN. Thus, removing overhangs with MBN requires enzyme titration ${ }^{[23]}$. To determine the best approach for blunting an overhang end, we tested those enzyme reactions on plasmids which, when digested by one enzyme, blunted to remove the overhang and ligated, would generate a different enzyme site. One plasmid (pCZV-bGHpA) was tested for 3' overhang (ApaI > PstI) and another (pCAG-dsRed) for 5' overhang (HindIII > NcoI) (see Figure 2B). With the 3' overhang, both Klenow fragments and MBN can generate a blunted DNA end, but Klenow is a better choice as it is easy and convenient to use. With the 5' overhang, MBN required titration to increase the yield of flushing DNA ends without altering the surrounding sequences.

In summary, we describe a simple and straightforward approach to generate a removable and scarless tSM by using the non-cutters RE sites with sticky ends. Not only does such tSM utilize convenient and powerful positive selection, but also no specific tools are required but routine enzyme manipulation.

\subsection{Making a unique site}

Ampk $\gamma$ is a component of energy sensor AMPK (AMP-activated protein kinase) in the cells. A point mutation (Arginine $965>$ Glutamine) has been proposed to pheno-copy the mutation of the AMPK $\alpha$ gene, which caused neurite swelling in flies ${ }^{[24]}$. Plasmid pUAST contains a Gal4 promoter, a traceable white gene, and a P element to generate a fly transgene (see Figure 3A). Together with Ampky: GFP, pUAST_loe_gfp_LK plasmid has no suitable RE sites around the desired mutation. Thus, it is troublesome to perform mutagenesis by PCR or by shuffling a small manageable fragment. We tested the approach of generating a point mutation in a BAC with this plasmid initially, using 50mer and gene specific primers to generate a targeting cassette with a mutation and RPSL-Neo. The mutation was confirmed and imported together with Neo selection after targeting. However, when RPSL was used for counter-selection with DNA fragments of either 40 mer oligos or longer generated by OL-PCR, the whole construct was recombined and lost PCR target (data not shown). This is more likely due to either instability of the priming plasmid or by failure of counter-selection.

As targeting worked precisely to bring in the mutation and SM, different approaches were required to remove SM. We decided to change the nearby StuI (32 bp from mutation site) to make it unique. The same OL-PCR product with the desired mutation (CGA $>$ CAA) that was used for reversing RPSL was TA cloned and the unique StuI site was digested and ligated with blunted AscI flanked em7-Neo (see Figure 3A). This ligation reconstituted the AscI site. After targeting, the mutation was confirmed ( 4 of 4). The resulting plasmid was digested with AscI to remove the Neo, and MBN was used to remove the added 5' overhang CGCG and restore the StuI site following ligation (see Figure 3B).

As MBN was found to be less effective in removing GC overhangs, a MBN titration was performed in order to determine the best conditions that produce DNA with StuI site restored ${ }^{[22]}$. The plasmids from titrated reactions that StuI can partially cut were transformed. To screen for those StuI restored plasmids, individual colonies ${ }^{[24]}$ were PCR with primer $\mathrm{L} / \mathrm{R}$ and the resulting PCR products were digested with or without StuI (see Figure 3C). Miniprep was then performed with 4 of 6 colonies with positive signals and digested with StuI. All were identical to that of the original plasmid but the mutation (see Figure 3C).

\subsection{Simplify targeting cassette making}

Four individual mutations were designed to study the function of FMR1 protein ${ }^{[25]}$. As a whole plasmid is less than $9 \mathrm{~kb}$ in size, the Quick Change (HF-PCR with 40 mer mutated oligos ${ }^{[26]}$ ) approach is well equipped to handle such a task. After multiple rounds of PCR reactions, no colonies were generated after transformations. The parental plasmid is likely pGFPC1 (Clontech) as it is a fusion between eGFP and mouse FMR1 and can be selected by Kan/Neo. After restriction enzyme diagnostics pattern didn't agree with the predicted results, DNA sequence reaction was performed to exam its flanking sequence. A second modification was realized to replace CMV with human FMR1 promoter (see Figure 4B). Without sequencing the whole plasmid to figure out why the Quick Change PCR reaction had difficulty with such plasmid, 
we decided to use targeting to generate each mutation. The benefits with such approaches are two-fold: first, only DNA sequence in targeted range is required, i.e. mFMR1 cDNA, and second, positive selection can circumvent the instability issue this plasmid might have.

Given that recombineering with $\lambda$-red requires only 30 50 of each homologous arm, and mutations between two homologous arms, whether it is a few nucleotide alterations, or additions from a few nucleotides to a whole transgene, all mutations can be integrated into the targeted plasmid. We designed a PCR-based approach to generate targeting cassettes with long oligos, which contain desired mutations, RE sites to enable selection marker removal without a scar, and specific primers to amplify selection markers. To modify FMR1, as NheI (GetagC) can't digest this plasmid, it became the enzyme of choice to flank selection markers (Zeocin R). With all the mutation sites, a nearby GC di-nucleotide was marked and 32 nts WT homologous arm and 38 nts arm with desired mutation and 5 nucleotides in 3' end of oligo was added to generate NheI/BtmI sites followed by selection marker specific sequence (see Figure 4A). After normal PCR reactions with Taq polymerase, PCR products were checked and column purified to be used as a targeting cassette in the recombineering reaction (DY380) (see Figure 4B). The targeted plasmid was digested with BmtI to remove SM and blunted by Klenow, and left mutations without altering other parts of plasmid (see Figure 4B). After repeating the same manipulation three more times, we successfully generated all four desired mutations.

A
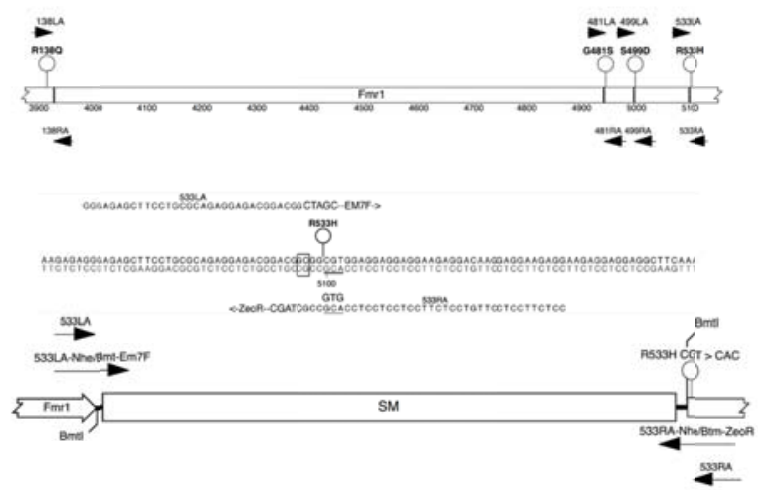

B
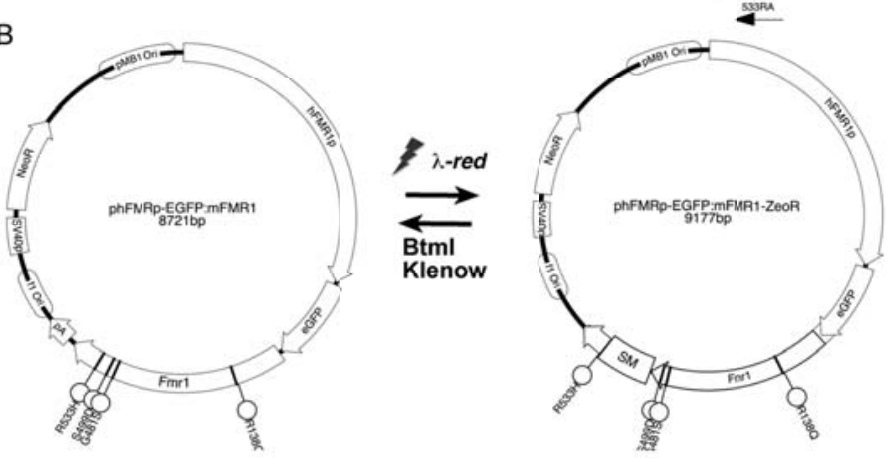

Figure 4. Streamlined targeting cassette generation. A) Targeting sites, arms and tSM. Several individual mutations (R130G, G481S, S499D and R533H) are required in mFMR1 cDNA. Diagram shows the location of mutations and targeting arms (LA/ RA $40 \mathrm{nts}$ each). Using R533H as an example, the sequence of homologous arms, insertion site (GC) and oligo sequences are listed. 533LA-Nhe/Bmt- Em7F primer contains left arm, NheI site and SM specific primer, and 533RA-Nhe/Bmt-ZeoR contains right homologous arm, mutation, NheI site and SM specific primer. Targeting cassette with tSM can be generated in one PCR reaction. B) Map of plasmids: Acceptor plasmid with presumption DNA sequence (phFMRp-EGFP-mFMR1) demonstrated the mutation sites (pin) and targeting sites (bar). After targeting, tSM (Zeo) was inserted together with R533H mutation to become phFMRp-EGFP-mFMR1-ZeoR. After BtmI digestion and Klenow blunting, tSM and NheI/BtmI sites were removed, and then plasmid was reversed to the original format with desired mutation. 


\subsection{In-frame fusion}

The idea of transitory selection markers opens the possibility that any DNA fragment can be tagged to enable it to become traceable. Such tagged fragments can be advantageous during complicated DNA engineering. After adding homologous arms, such traceable DNA can become a targeting cassette. Not only can it be used to bring in point mutations, but also for peptide tagging or CDS fusion.

As a self-splicing peptide derived from the Foot and Mouth Disease virus, F2a peptide allows one CDS to express more than 1 protein from a single transcript ${ }^{[27,28]}$. Here, an F2a peptide is inserted to break up the fusion protein eGFP-Cre (see Figure 5A), which needs to be in frame both with eGFP and Cre ${ }^{[29]}$. From a GGCC tetra-nucleotide within the F2a DNA, we added CCGG in between to create an FseI site to tag the F2a fragment with a tSM for targeting. F2a has 24 amino acids with $72 \mathrm{bp}$, which can be completely covered with 2 long primers. One oligo contains the N-terminal sequence of F2a, FseI and primer for em7 promoter. Another oligo contains the C-terminal of F2a, FseI and primer for Neo (see Figure 5B). HF-PCR was performed on em7Neo template to make F2a-FseI-em7Neo. A second PCR with primers containing GFPC-F2aN or CreN-F2aR was used to generate the targeting cassette. By recombineering, GFP-Cre was separated by F2a with selection, which was then removed by FseI digestion and blunted with the Klenow reaction. DNA sequencing confirmed the insertion of the F2a peptide in frame with both eGFP and Cre.

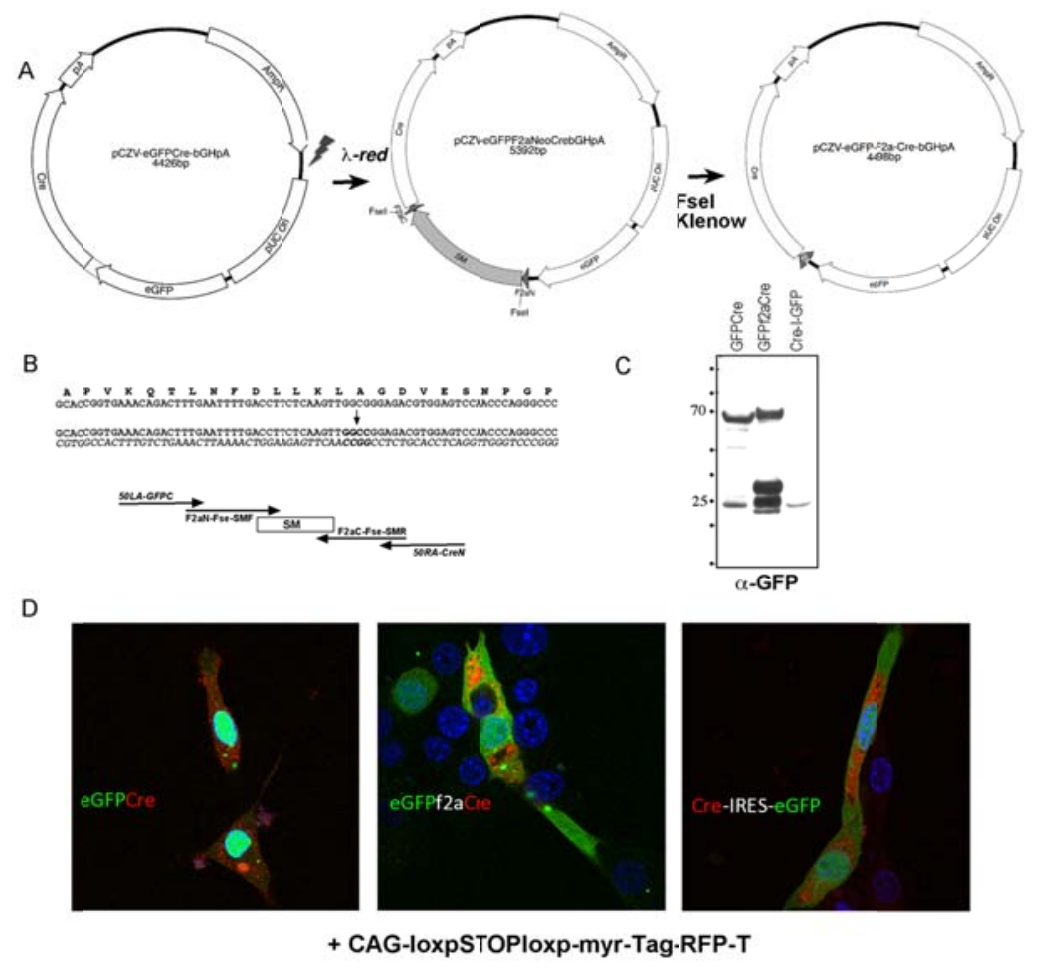

Figure 5. Fused 2a peptide. A) Map of Plasmids: Accepter plasmid containing eGFP-Cre was fused with targeting cassette with F2a and tSM after targeting. tSM can then be removed, leaving in-frame fused F2a. B) Native F2a sequence and assembling a targeting cassette by PCR. C) Protein blot stained with Anti-GFP antibody with protein extract after transfection to NIH3T3 cells with pCAG-eGFP-Cre, pCAG-eGFP-F2a-Cre and pCAG-Cre-IRES-eGFP plasmids respectively. D. Co-transfect with Cre reporter plasmids (pCAG-LoxP-STOP-myr-tagRFP-T) to assay function of Cre and GFP distribution in the cells. The cell nucleus was counter-stained with DAPI (blue) to mark cells as well as the location of the nucleus.

Since the function of eGFP, F2a and Cre can be assayed; eGFP-F2a-Cre and its parental fusion construct eGFP-Cre were cloned into an expression vector (pCAG). Together with Cre-IRES-eGFP as a control, all three vectors were transfected 
into NIH3T3 cells respectively by lipofection. Total protein extract was prepared and a protein blot was performed with anti-GFP antibody (see Figure 5C). The IRES-eGFP showed a band $27 \mathrm{kD}$, which is the correct size of GFP. GFP-Cre fusion protein is around $68 \mathrm{kD}$ with a processed product that has the same size of native GFP. Multiple bands were detected with eGFP-F2a-Cre transfection. The large protein is likely the GFP-F2a-GFP fusion protein, as well as several processed proteins, eGFP-F2a and eGFP, which is consistent with F2a properties. The F2a self-splicing efficiency is more than $70 \%$ when compared to the intact and processed bands.

To assay Cre function, the expression constructs were respectively co-transfected with pCAG-STOP-myr-tagRFP-T plasmid into mammalian cells, and Cre can be monitored by the expression of tag-RFP-T (red) ${ }^{[30]}$. With myristoylation, myr-tagRFP-T is expected to localize on the membrane. After analyzing confocal images, the major red color is associated with ER (see Figure 5D) in all three constructs, suggesting Cre is able to remove loxP floxed STOP cassette. Due to its size, cytosolic GFP is expressed in the whole cell, and eGFP-Cre is localized in the nucleus. When GFP and Cre were separated with F2a, most double-staining cells with red and green colors exhibited cytoplasmic distribution of GFP and only a few were in the nucleus, which is consistent with the protein blot result (see Figure 5D).

\section{Discussion}

Recombineering has been used to generate site-directed mutagenesis without leaving extra-DNA (scar) in a BAC as well as in the bacterial genome by using oligos with mutations to serve as primers for DNA replication ${ }^{[7,10]}$. Without a proper way to distinguish mutated from parental plasmids, substantial screening efforts are necessary. Cassettes such as Tet R, GalK, Neo-SacB, and RPSL-Neo, can be both positively and negatively selected that have been used to facilitate the identification of recombined DNA ${ }^{[11]}$. Positive selection markers provide an easy way to "tag" mutated DNA after targeting. Counter-selection can then be applied to isolate the loss of selection markers after primer priming. However, due to the special requirements such as special strains of bacteria (rpsl, galk) and the high rate of mutation, counter selection is more suitable with low copy and large plasmids such as BAC. In addition, mismatched priming with a mutated oligo can yield off-site targets. As such, this approach is limited to making point mutations. To solve those issues and to take advantage of strand invasion and its tolerance of minor mismatches in homologous recombination, a single positive selection marker which can provide scarless removal was employed to introduce mutations in acceptor plasmids through targeting.

Theoretically, placing a selection marker inside an intron or intein can achieve the "scarless" requirement through relying on cellular machineries to remove the selection marker ${ }^{[31,32]}$. However, both introns and inteins can work only with protein coding region, and intein can only be used in between domains. As for introns, additional sequences in exons and introns are needed for precise splicing, thus limiting their usage in carrying selection markers for targeting. The need to find a non-cutter will be relieved when an enzyme recognizes a rare DNA sequence, which can be removed by either such an enzyme or together with other enzymes. Development of zinc-finger nuclease (ZFN), TAL effector nuclease (TALEN) and Cas9/Guide RNA indicates a possible artificial endonuclease with a long recognition site, which could be unique within the human genome ${ }^{[33-35]}$. With its current design, artificial endonuclease is similar to a meganuclease (homing enzyme), which recognizes a long stretch of DNA, but its recognition site can't be removed easily after digestion. It is possible to either tweak the artificial endonuclease arrangement to meet the "transitory" criteria or to combine homologous recombination repair as used with a meganuclease ${ }^{[36]}$. Recently, transposases have yielded no excision footprint, which may provide a way to generate a tSM ${ }^{[37]}$. PiggyBac recognition sites flanking selection markers have been successful in repairing tyrosinase point mutations after ES cell targeting. Subsequently, they can be removed with PiggyBac transposase to resume an original open reading frame ${ }^{[38]}$. The limitation is that PiggyBac requires a TTAA site for insertion and excision and whether such reactions can be used in genetic engineering is unknown.

Combining the benefit of recombineering with enzymatic manipulation, we devised a seamless and effective way of making site-directed mutagenesis. A few examples of DNA fabrications were presented to support the usefulness of our methodologies. Not only can we modify plasmids with point mutations, but we can also enable DNA recombination to 
bring in mutations or generate fusion proteins. Different from PCR and BAC-based site-directed mutagenesis, our method is not limited to generating point mutations, but also includes importing or removing DNA as truncation. Using a "transitory" selection marker, one can tag DNA for complicated manipulation such as Epitope tags or fusion proteins. With existing mutations, after tagging mutated DNA with tSM, it can be shuttled around via ligation or targeting as DNA itself can be the homologous arm. Although our procedure requires RE manipulation, it is not limited by location, the availability of RE sites, nor addition of restriction site, but it is a rare occasion that a non-cutter can be useful. With the efficiency of targeting and the transient nature of tSM, it is conceivable that high throughput site-directed mutagenesis is possible to make a slight difference in otherwise identical plasmids, or by making random mutations in designated areas to generate pools of mutations in a defined domain ${ }^{[39]}$. Indeed, based on our experience, as long as homologous arms can form a stable complex with acceptor plasmids, single, double or even multiple mutations can be imported in one targeting event.

\section{Acknowledgments}

Members in the Neuroscience Center at UNC-CH requested the modification of individual constructs. Dr. McGarry MS provided manuscript preparation and editing services. BAC Engineering and Confocal Core facilities are funded by a Center Grant (P30 NS045892-05) to the UNC-Neuroscience Center from National Institute of Neurological Disorders and Stroke.

\section{References}

[1] Hutchison CA 3rd, Phillips S, Edgell MH, et al. Mutagenesis at a specific position in a DNA sequence. J Biol Chem. 1978; 253(18): 6551-60. PMid:681366.

[2] Braman J, Papworth C, Greener A. Site-directed mutagenesis using double-stranded plasmid DNA templates. Methods Mol Biol. 1996; 57: 31-44. http://dx.doi.org/10.1385/0-89603-332-5:31

[3] Kunkel TA. Rapid and efficient site-specific mutagenesis without phenotypic selection. Proc Natl Acad Sci USA. 1985; 82(2): 488-92. PMid:3881765. http://dx.doi.org/10.1073/pnas.82.2.488

[4] Weiner MP, Costa GL, Schoettlin W, et al. Site-directed mutagenesis of double-stranded DNA by the polymerase chain reaction. Gene. 1994; 151:119-23. http://dx.doi.org/10.1016/0378-1119(94)90641-6

[5] Copeland NG, Jenkins NA, Court DL. Recombineering: a powerful new tool for mouse functional genomics. Nat Rev Genet. 2001; 2: 769-79. PMid:11584293. http://dx.doi.org/10.1038/35093556

[6] Ellis HM, Yu D, DiTizio T, et al. High efficiency mutagenesis, repair, and engineering of chromosomal DNA using singlestranded oligonucleotides. Proc Natl Acad Sci USA. 2001; 98: 6742-6. PMid:11381128. http://dx.doi.org/10.1073/pnas.121164898

[7] Muyrers JP, Zhang Y, Testa G, et al. Rapid modification of bacterial artificial chromosomes by ET-recombination. Nucleic Acids Res. 1999; 27: 1555-7. PMid:10037821. http://dx.doi.org/10.1093/nar/27.6.1555

[8] Zhang Y, Buchholz F, Muyrers JP, et al. A new logic for DNA engineering using recombination in Escherichia coli. Nat Genet. 1998; 20: 123-8. PMid:9771703. http://dx.doi.org/10.1038/2417

[9] Noll S, Hampp G, Bausbacher H, et al. Site-directed mutagenesis of multi-copy-number plasmids: Red/ET recombination and unique restriction site elimination. BioTechniques. 2009; 46: 527-33. PMid:19594452. http://dx.doi.org/10.2144/000113150

[10] Murphy KC. Use of bacteriophage lambda recombination functions to promote gene replacement in Escherichia coli. J Bacterial. 1998; 180: 2063-71. PMid:9555887.

[11] Yang Y, Sharan SK. A simple two-step, hit and fix method to generate subtle mutations in BACs using short denatured PCR fragments. Nucleic Acids Res. 2003; 31: e80. PMid:12888532. http://dx.doi.org/10.1093/nar/gng080

[12] Reyrat JM, Pelicic V, Gicquel B, et al. Counterselectable markers: untapped tools for bacterial genetics and pathogenesis. Infect Immun. 1998; 66: 4011-7. PMid:9712740.

[13] Warming S, Costantino N, Court DL, et al. Simple and highly efficient BAC recombineering using galK selection. Nucleic Acids Res. 2005; 33: e36. PMid:15731329. http://dx.doi.org/10.1093/nar/gni035

[14] Gibson DG, Young L, Chuang RY, et al. Enzymatic assembly of DNA molecules up to several hundred kilobases. Nature Methods. 2009; 6 (5): 343-5. PMid:19363495. http://dx.doi.org/10.1038/nmeth.1318 
[15] Poteete AR. Involvement of DNA replication in phage Lambda red-mediated homologous recombination. Mol Microbiol. 2008; 68(1): 66-74. PMid:18333884. http://dx.doi.org/10.1111/j.1365-2958.2008.06133.x

[16] Bouvier J, Cheng JG. Recombineering-based procedure for creating Cre/loxP conditional knockouts in the mouse. Curr Protoc Mol Biol. 2009; Chapter 23: Unit 23.13.

[17] Hollenback SM, Lyman S, Cheng JG. Recombineering-based procedure for creating BAC transgene constructs for animals and cell lines. Curr Protoc Mol Biol. 2011; Chapter 23: Unit 23.14. http://dx.doi.org/10.1002/0471142727.mb2314s95

[18] Maniatis T, Fritsch EF, Sambrook J. Molecular cloning: a laboratory manual 1982. Cold Spring Harbor, NY: Cold Spring Harbor Laboratory, p545.

[19] Garske AL, Peters U, Cortesi AT, et al. Chemical genetic strategy for targeting protein kinases based on covalent complementarity. Proc Natl Acad Sci USA. 2011; 108: 15046-52. PMid:21852571. http://dx.doi.org/10.1073/pnas.1111239108

[20] Ho SN, Hunt HD, Horton RM, et al. Site-directed mutagenesis by overlap extension using the polymerase chain reaction. Gene. 1989; 77:51-9. http://dx.doi.org/10.1016/0378-1119(89)90358-2

[21] Johnson PH, Laskowski M Sr. Mung bean nuclease I. II. Resistance of double stranded deoxyribonucleic acid and susceptibility of regions rich in adenosine and thymidine to enzymatic hydrolysis. J Bio Chem. 1970; 245: 891-8.

[22] Mikulski AJ, Laskowski M Sr. Mung bean nuclease I III. Purification procedure and (3') omega monophosphatase activity. J Bio Chem. 1970; 245: 5026-31.

[23] Sharp AJ, Slater RJ. Mung Bean Nuclease I (EC 3.1.30.1), in Enzymes of Mol Biol. M.M. Burrell. Humana Press: Totowa, NJ. 2013: 253-61.

[24] Mirouse V, Swick LL, Kazgan N, et al. LKB1 and AMPK maintain epithelial cell polarity under energetic stress. J Cell Biol. 2007; 177: 387-92. PMid:17470638. http://dx.doi.org/10.1083/jcb.200702053

[25] Verkerk AJ, Pieretti M, Sutcliffe JS, et al. Identification of a gene (FMR-1) containing a CGG repeat coincident with a breakpoint cluster region exhibiting length variation in fragile X syndrome. Cell. 1991; 65 (5): 905-14. http://dx.doi.org/10.1016/0092-8674(91)90397-H

[26] Zheng L, Baumann U, Reymond JL. An efficient one-step site-directed and site-saturation mutagenesis protocol. Nucleic Acids Res. 2004 Aug 10; 32(14): e115. PMid:15304544. http://dx.doi.org/10.1093/nar/gnh110

[27] Ryan MD, King AM, Thomas GP. Cleavage of foot-and-mouth disease virus polyprotein is mediated by residues located within a 19 amino acid sequence. Gen Virol. 1991; 72(Pt 11): 2727-32. PMid:1658199. http://dx.doi.org/10.1099/0022-1317-72-11-2727

[28] Kim JH, Lee SR, Li LH, et al. High cleavage efficiency of a 2A peptide derived from porcine teschovirus-1 in human cell lines, zebrafish and mice. PloS one. 2011; 6: e18556. PMid:21602908. http://dx.doi.org/10.1371/journal.pone.0018556

[29] Le Y, Miller JL, Sauer B. GFPcre fusion vectors with enhanced expression. Ana Biochem. 1999; 270: $334-6$. PMid:10334853. http://dx.doi.org/10.1006/abio.1999.4110

[30] Shaner NC, Lin MZ, McKeown MR, et al. Improving the photostability of bright monomeric orange and red fluorescent proteins. Nat Methods. 2008; 5: 545-51. PMid:18454154. http://dx.doi.org/10.1038/nmeth.1209

[31] Poser I, Sarov M, Hutchins JRA, et al. BAC TransgeneOmics: a high-throughput method for exploration of protein function in mammals. Nat Methods. 2008; 5: 409-15. PMid:18391959. http://dx.doi.org/10.1038/nmeth.1199

[32] Ramsden R, Arms L, Davis TN, et al. An intein with genetically selectable markers provides a new approach to internally label proteins with GFP. BMC Biotechnol. 2011; 11: 71. PMid:21708017. http://dx.doi.org/10.1186/1472-6750-11-71

[33] Kim YG, Cha J, Chandrasegaran S. Hybrid restriction enzymes: zinc finger fusions to Fok I cleavage domain. Proc Natl Acad Sci USA. 1996; 93: 1156-60. PMid:8577732. http://dx.doi.org/10.1073/pnas.93.3.1156

[34] Christian M, Cermak T, Doyle EL, et al. Targeting DNA double-strand breaks with TAL effector nucleases. Genetics. 2010; 186: 757-61. PMid:20660643. http://dx.doi.org/10.1534/genetics.110.120717

[35] Ran FA, Hsu PD, Wright J, et al. Genome engineering using the CRISPR-Cas9 system. Nat Protoc. 2013 ; 8 (11): $2281-308$. PMid:24157548. http://dx.doi.org/10.1038/nprot.2013.143

[36] Blank K, Hensel M, Gerlach RG. Rapid and highly efficient method for scarless mutagenesis within the Salmonella enterica chromosome. PloS one. 2011; 6: e15763. PMid:21264289. http://dx.doi.org/10.1371/journal.pone.0015763

[37] Ni J, Clark KJ, Fahrenkrug SC, et al. Transposon tools hopping in vertebrates. Briefings Funct Genomics Proteomics. 2008; 7: 444-53. PMid:19109308. http://dx.doi.org/10.1093/bfgp/eln049

[38] Yusa K, Rashid ST, Strick-Marchand H, et al. Targeted gene correction of alpha1-antitrypsin deficiency in induced pluripotent stem cells. Nature. 2011; 478: 391-4. PMid:21993621. http://dx.doi.org/10.1038/nature10424

[39] Warner JR, Reeder PJ, Karimpour-Fard A, et al. Rapid profiling of a microbial genome using mixtures of barcoded oligonucleotides. Nature Biotechnol. 2010; 28: 856-62. PMid:20639866. http://dx.doi.org/10.1038/nbt.1653 